\title{
Recuperação de Solventes Orgânicos de Laboratório. Alternativas Econômicas e Ecologicamente Adequadas
}

\author{
Bruna C. Dias, Filipe W. Schwarz \& Eduardo R. de Oliveira
}

\begin{abstract}
A UFRGS possui mais de 250 laboratórios que produzem resíduos químicos. Gerando uma quantidade anual de mais de 15 mil litros de misturas de solventes orgânicos. O descarte de forma adequada e segundo as normas ambientalmente aceitas é caro, na medida em que no Rio Grande do Sul não é permitida a incineração e nem o co-processamento. Este trabalho de pesquisa tem como objetivo desenvolver métodos de separação, tratamento e recuperação de solventes orgânicos, visando à purificação dos mesmos, para estes serem reutilizados, evitando gastos na compra de novos reagentes e no descarte dos mesmos. Sua metodologia geral consiste em trabalhar inicialmente em pequena escala e após estabelecido o melhor método de recuperação, passando o processo para grande escala. Foi possível recuperar muitos solventes de forma simples e com alto rendimento como hexano, clorofórmio, metanol, diclorometano, acetato de etila, xilol e etanol. Em alguns casos não é preciso a separação dos solventes, podendo estes serem reutilizados na forma de mistura como é o caso do acetato de etila e hexano. Este projeto também está sendo implementando como projeto piloto no setor de química orgânica da graduação, e futuramente pretende-se expandir para mais setores da universidade, sendo então um método econômico e sustentável.
\end{abstract}

\section{Palavras Chave: Recuperação; Solventes Orgânicos; Resíduo; Ambiental.}

The UFRGS has more than 250 laboratories that produce chemical waste. Generating an annual amount of more than 15 thousand liters of mixtures of organic solvents. Disposal in an adequate manner and according to the environmentally accepted standards is expensive, insofar as incineration and co-processing are not allowed in Rio Grande do Sul. This research aims to develop methods of separation, treatment and recovery of organic solvents, in order to purify them, to be reused, avoiding expenses in the purchase of new reagents and in the disposal of them. Its general methodology is to work initially on a small scale and after establishing the best method of recovery, passing the process to a large scale. Many solvents could be recovered simply and in high yields such as hexane, chloroform, methanol, dichloromethane, ethyl acetate, xylene and ethanol. In some cases it is not necessary to separate the solvents, which can be reused in the form of a mixture as is the case with ethyl acetate and hexane. This project is also being implemented as a pilot project in the undergraduate organic chemistry sector, and in the future it is intended to expand to more sectors of the university, being an economical and sustainable method.

Keywords: Recovery; Organic Solvents; Waste; Environmental. 


\section{Introdução}

Atualmente existe uma grande preocupação com a poluição do meio ambiente, um dos fatores que aumenta a poluição do ar é a incineração que consiste num processo de decomposição térmica, muito utilizado para o descarte do lixo e resíduos químicos. Neste processo pode ocorrer a formação de substâncias tóxicas e nocivas como as dioxinas e furanos.

A Universidade Federal do Rio Grande do Sul (UFRGS) possui mais de 250 laboratórios que produzem resíduos químicos. Dentre esses resíduos tem-se uma quantidade anual de mais de 15 mil litros de misturas de solventes orgânicos de várias naturezas, halogenados e não halogenados. O descarte de forma adequada e segundos as normas ambientalmente aceitas, é problemático e caro, na medida que, no Rio Grande do Sul não é permitida a incineração e nem o co-processamento. Apenas em 2015 a UFRGS gastou cerca de $\mathrm{R} \$ 167.000,00$ para enviar cerca de, 18 toneladas de solventes orgânicos para incineração no RJ, já em 2016 a UFRGS gastou, cerca de, R\$50.000,00 para envio de 15.7 toneladas de solventes orgânicos para incineração em SP.

O Centro de Gestão e Tratamento de Resíduos Químicos (CGTRQ) é um órgão auxiliar do Instituto de Química da UFRGS, responsável pela coleta e tratamento dos resíduos químicos provenientes dos laboratórios da Universidade, o qual desenvolveu um método interno de classificação dos resíduos químicos recebidos, conforme a Figura 1.

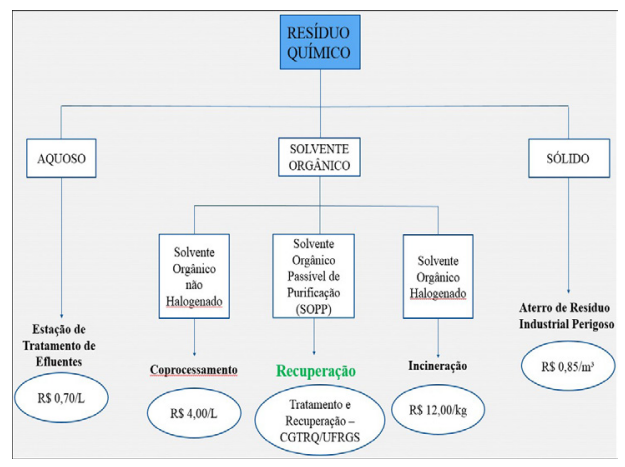

Figura 1. Classificação dos Resíduos usada no CGTRQ

\section{Metodologia}

Foi realizado um levantamento nos dados dos últimos dois anos de recebimento de resíduos do CGTRQ, a fim de localizar os geradores de maior volume de solventes orgânicos passíveis de purificação (SOPP) que são misturas de dois a três solventes, para então entrar em contato com esses laboratórios e desta forma tentar conscientizar os mesmos da importância da coleta seletiva.

A metodologia geral deste trabalho consiste em trabalhar primeiramente em pequena escala, com estudo de micro destilação e pré-tratamento, seguida pela análise dos resultados por espectros de infravermelho (IV) e ressonância magnética nuclear ( $\mathrm{RMN})$, índice de refração, densidade e cromatografia gasosa (CG). Com o objetivo de se estabelecer o melhor método de recuperação, de preferência de forma simples, com menor custo e gerando a menor quantidade de resíduo possível. Depois de desenvolvido um método, passa-se então o processo para grande escala com destilador do tipo Spinning band semi-automatizado.

Acetato de Etila (AcOEt): Foi recebido 20L da mistura de acetato de etila e água, utilizada em extrações de alcaloides, proveniente de laboratórios de farmacognosia. A formação de azeótropo exigiu prétratamento com secante. Foi testada a eficiência dos secantes $\mathrm{MgSO}_{4}$, e $\mathrm{CaCl}_{2}$, para determinar qual o melhor secante relacionando eficiência e preço do reagente, sendo que o apresentou essas características foi o $\mathrm{CaCl}_{2}$, e por destilação, obteve-se $73 \%$ de AcOEt puro, cujas análises por CG, infravermelho e RMN $1 \mathrm{H}$ confirmam a pureza. Conforme mostram a Figura 2, a fração 1, em que se pode observar a banda de estiramento $\mathrm{OH}$ da água e na Figura 3, fração 2 majoritária onde podemos confirmar a ausência de água.

\section{SOPPS}

Na Tabela 1, encontra-se o resultado da recuperação de SOPPs com apenas um solvente, que é o caso do hexano, clorofórmio e metanol, provenientes de locais diferentes. 


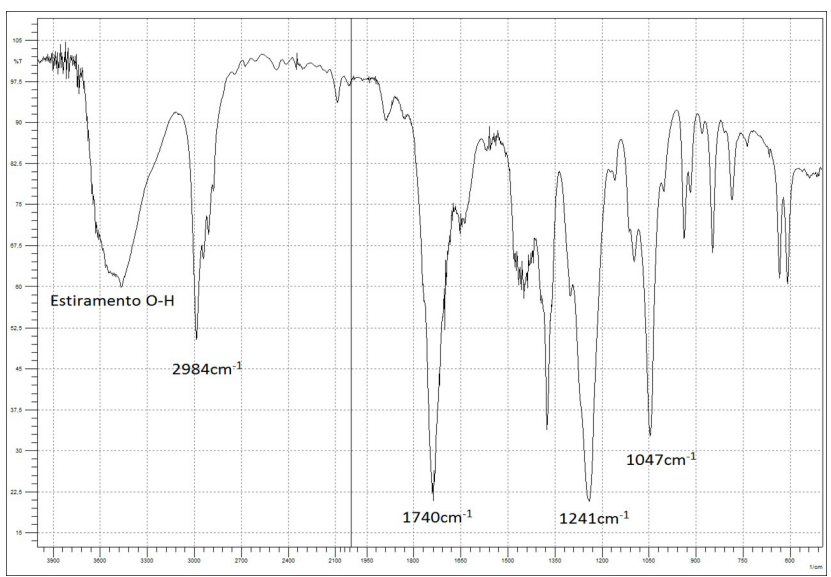

Figura 2. Espectro de infravermelho de amostra de acetato de etila e água.

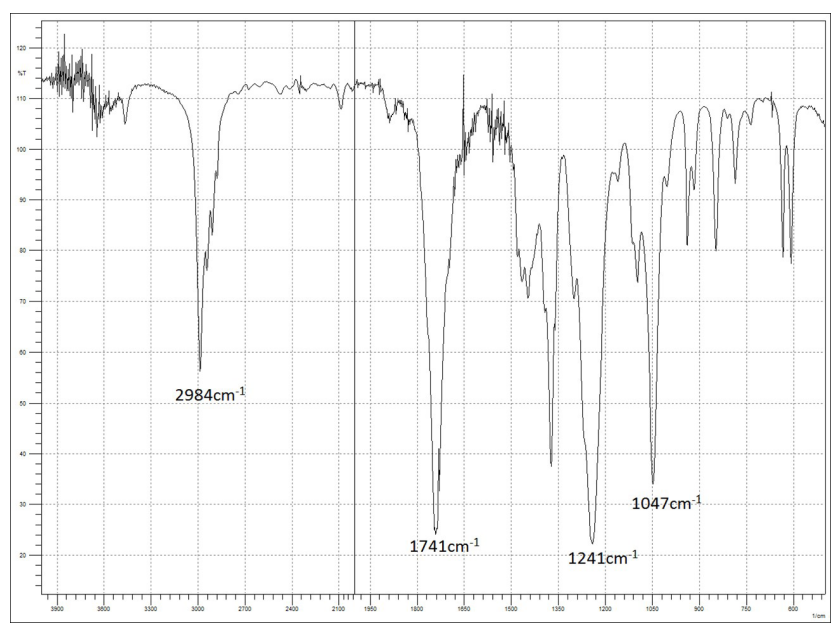

Figura 3. Espectro de infravermelho de amostra de acetato de etila, após secagem e destilação.

Com exceção do clorofórmio que precisou previamente de uma separação de fases, não precisaram de pré-tratamento e com apenas uma destilação podem ser recuperados e assim reutilizados com altos rendimentos, confirmado esses resultados por $\mathrm{CG}$.
Tabela 1. Resultados de SOPPs com apenas um solvente.

\begin{tabular}{|c|c|c|}
\hline Entradas & Origem & $\begin{array}{c}\text { Rendimento (\% } \\
\text { volume) }\end{array}$ \\
\hline hexano & Empresa & 94 \\
\hline clorofórmio & Empresa & 95 \\
\hline metanol & $\begin{array}{c}\text { Departamento de } \\
\text { Físico-química }\end{array}$ & 96 \\
\hline
\end{tabular}

Xilol: O CGTRQ recebe todos os anos mais de 100 litros da mistura de xilenos, etanol, água, corante e parafina, provenientes de laboratórios de patologia da UFRGS. O processo de recuperação não precisa de nenhum tratamento prévio, e já foi otimizado em grande escala, por CG percebeu-se que as primeiras frações consistem em mistura de etanol e xilenos, mas com temperatura acima de $130{ }^{\circ} \mathrm{C}$ só destila xilol, conforme a Tabela 2.

Tabela 2. Resultados da destilação da amostra de xilol.

\begin{tabular}{|c|c|c|c|c|}
\hline Fração & $\begin{array}{c}\text { Temperatura } \\
{ }^{\circ} \mathrm{C}\end{array}$ & $\begin{array}{c}\% \text { Vol. } \\
\text { Obtido }\end{array}$ & $\begin{array}{c}\% \text { Área } \\
\text { picos } \\
\text { etanol }\end{array}$ & $\begin{array}{c}\text { \% Área } \\
\text { picos xilol }\end{array}$ \\
\hline 1 & 23,8 a 130.9 & 23,3 & 57,8 & 42,2 \\
\hline 2 & 131,4 a 138,9 & 76,7 & 0 & 100 \\
\hline
\end{tabular}

Com comparações dos resultados em CG e RMN determinou-se as proporções entre os isômeros, conforme Figura 4.

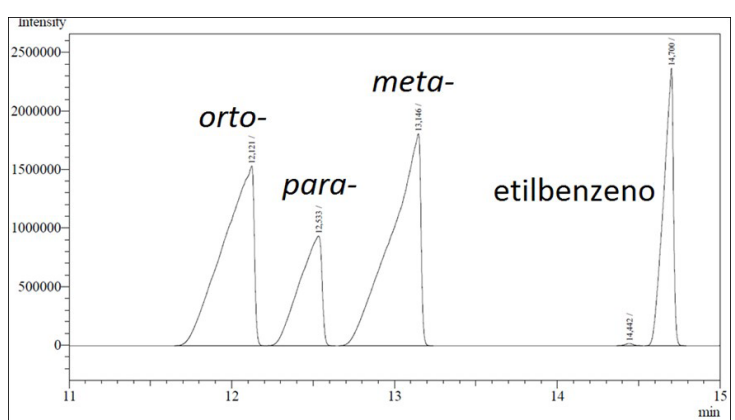

Figura 4. Cromatograma de amostra de xilol destilado com seus respectivos isômeros. 
Já foram devolvidos mais de 200 litros aos laboratórios de origem para serem reutilizados, confirmando a eficiência da recuperação.

Acetato de Etila e hexano: A mistura de acetato de etila e hexano é muito utilizada nos laboratórios de química para colunas cromatográficas. Não é viável separar um solvente do outro por destilação. Neste caso, foi obtida uma curva de calibração utilizando misturas dos padrões dos dois solventes no cromatógrafo o qual possibilita a determinação da concentração da mistura de AcOEt e hexano (após destilação para retirar impurezas). A separação da mistura não é necessária devido à sua reutilização, sob forma de mistura, desde que padronizada. Realizamos testes para validação da curva utilizando diferentes misturas de hexanos (um comercial e outro recuperado) utilizando micropipetadores para reduzir erros. Os dados se encontram na Tabela 3.

Tabela 3. Tabela dos resultados do teste da curva padrão de acetato de etila em hexano.

\begin{tabular}{|c|c|c|c|}
\hline Amostra & \% volumétrica & $\begin{array}{c}\text { \% calculada } \\
\text { pela curva }\end{array}$ & $\begin{array}{c}\text { Diferença } \\
\text { medida }\end{array}$ \\
\hline $\begin{array}{c}\text { AcOEt em } \\
\text { hexano } \\
\text { recuperado }\end{array}$ & 23 & 24,73 & $+1,75$ \\
\hline $\begin{array}{c}\text { AcOEt em } \\
\text { hexano } \\
\text { comercial }\end{array}$ & 23 & 25,21 & $+2,21$ \\
\hline $\begin{array}{c}\text { AcOEt em } \\
\text { hexano } \\
\text { recuperado }\end{array}$ & 55 & 54,06 & $-0,94$ \\
\hline $\begin{array}{c}\text { AcOEt em } \\
\text { hexano } \\
\text { comercial }\end{array}$ & 55 & 56,16 & $+1,16$ \\
\hline $\begin{array}{c}\text { AcOEt em } \\
\text { hexano } \\
\text { recuperado }\end{array}$ & 78 & 76,52 & $-1,48$ \\
\hline $\begin{array}{c}\text { AcOEt em } \\
\text { hexano } \\
\text { comercial }\end{array}$ & 78 & 77,95 & $-0,05$ \\
\hline
\end{tabular}

Vemos pela tabela que a análise em CG (após correção) mostrou composições apenas levemente diferentes das medidas na pipeta, validando a curva de calibração. Tendo em vista a reutilização em novas colunas cromatográficas, a diferença de até 3 unidades na concentração medida não deve influenciar muito no processo e, portanto a curva pode ser utilizada. Realizou-se então a destilação em grande escala de duas amostras de misturas dos solventes, provenientes de laboratórios de pesquisas diferentes, obtendo-se em uma fração única de 4 litros da mistura com $59 \%$ de AcOEt e no segundo caso uma fração única de 2 litros com 40\% de AcOEt calculada pela curva., ambas retornaram aos laboratórios de origem para serem reutilizadas.

\section{ETANOL COM POLPA DE CAQUI}

O CGTRQ recebeu da Faculdade de Agronomia uma mistura de etanol com polpa de caqui, e com apenas uma destilação foi possível recuperar $90 \%$ do volume de etanol aquoso, confirmado esse valor pelo CG no qual só aparece o pico do etanol conforme mostra na Figura 5, como o mesmo será reutilizado com frutas, não havia a necessidade de secagem, então após a determinação do teor de água por índice de refração e densidade, foram entregues 32 litros para os laboratórios de origem para serem reutilizados.

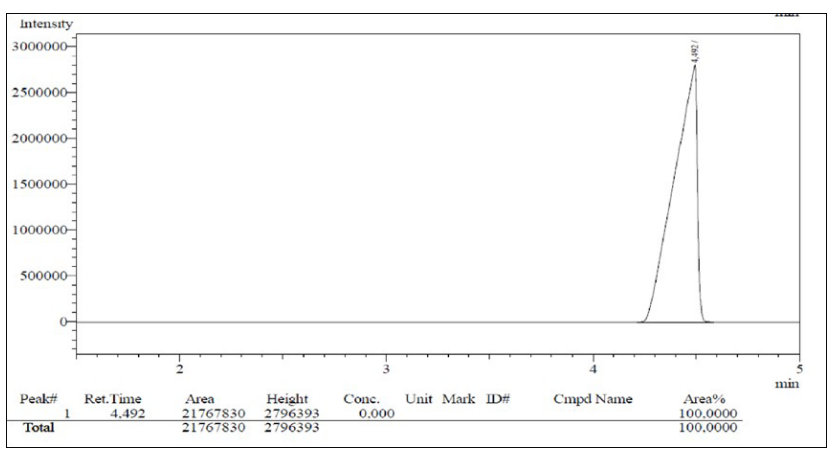

Figura 5. Cromatograma etanol com polpa de caqui após destilação. 
Acetonitrila: O CGTRQ recebe mensalmente mais de 10 litros da mistura de acetonitrila, metanol e água, proveniente da Faculdade de Farmácia, que a utiliza em análises de cromatografia líquida de alta eficiência (HPLC), cujo descarte é caro, sendo a acetonitrila um reagente com alto valor agregado. Trabalhou-se com 8 amostras diferentes desse tipo de resíduo, oriundos de laboratórios diferentes em uma dessas amostras não havia especificação da proporção de água, nos casos de amostras muito aquosas ou sem especificação no rótulo, foi realizado uma separação de fases por criogenia, após destilação, observou-se que esse resíduo tinha 4 perfis diferentes como acetonitrila, metanol e água, ou $\mathrm{CH}_{3} \mathrm{CN}$ e $\mathrm{MeOH}$, ou $\mathrm{CH}_{3} \mathrm{CN}$ e $\mathrm{CH} 2 \mathrm{Cl}_{2}$ ou somente $\mathrm{CH}_{3} \mathrm{CN}$. Nos casos das amostras que continham $\mathrm{MeOH}$ e/ou $\mathrm{CH}_{2} \mathrm{Cl}_{2}$ foi realizado um pré-tratamento com $\mathrm{NaOH}$, Foi observado também que na maioria dos casos, o que está descrito no rótulo não condiz com o que está realmente na garrafa de resíduo, como pode ser observado na Tabela 4.

Tabela 4. Resultados das amostras de resíduo de acetonitrila proveniente de resíduo de HPLC.

\begin{tabular}{|c|c|c|c|}
\hline Entradas & $\begin{array}{l}\text { Descrição do } \\
\text { Rótulo }\end{array}$ & $\begin{array}{l}\text { Volume } \\
\text { (L) }\end{array}$ & Resultado \\
\hline 1 & $\begin{array}{c}\mathrm{CH}_{3} \mathrm{CN}, \mathrm{MeOH} \mathrm{e} \\
\mathrm{H}_{2} \mathrm{O}\end{array}$ & 5 & $\begin{array}{c}44 \% \text { aquoso, } \mathrm{CH} 3 \mathrm{CN} \\
98 \%, \mathrm{MeOH}_{0}, 6 \% \mathrm{e} \\
\mathrm{CH}_{2} \mathrm{Cl}_{2} 1,4 \%\end{array}$ \\
\hline 2 & $\begin{array}{c}\mathrm{CH}_{3} \mathrm{CN} \text { e } \mathrm{H}_{2} 0 \\
\quad(60: 40) \\
\end{array}$ & 5 & $\begin{array}{l}65 \% \text { aquoso, } \mathrm{CH} 3 \mathrm{CN} \\
98,7 \% \text { e } \mathrm{MeOH} 1,3 \%\end{array}$ \\
\hline 3 & $\begin{array}{c}\mathrm{CH} 3 \mathrm{CN} \text { e H2O } \\
(90: 10) \\
\end{array}$ & 5 & $\begin{array}{l}50 \% \text { aquoso, } \mathrm{CH}_{3} \mathrm{CN} \\
95,4 \% \text { e } \mathrm{CH}_{2} \mathrm{Cl}_{2} 4,6 \%\end{array}$ \\
\hline 4 & $\begin{array}{l}\mathrm{CH}_{3} \mathrm{CN} \text { e ácido } \\
\text { fórmico }(99: 1) \\
\end{array}$ & 0,5 & $\mathrm{CH}_{3} \mathrm{CN} 100 \%$ \\
\hline 5 & $\mathrm{CH}_{3} \mathrm{CN}$ P.A (antiga) & 0,3 & $\mathrm{CH}_{3} \mathrm{CN} 100 \%$ \\
\hline 6 & $\begin{array}{c}\mathrm{CH}_{3} \mathrm{CN}, \mathrm{MeOH} \\
(90: 10)\end{array}$ & 1 & Só MeOH \\
\hline 7 & $\begin{array}{c}\mathrm{CH}_{3} \mathrm{CN}, \mathrm{MeOH}, \\
\mathrm{H}_{2} \mathrm{O}(80: 10: 10) \\
\text { e } 0,01 \% \text { de ácido } \\
\text { trifluoacético }\end{array}$ & 4,5 & $\begin{array}{l}\mathrm{CH}_{3} \mathrm{CN} 97 \%, \mathrm{MeOH} \\
2,5 \% \text { e } \mathrm{CH} 2 \mathrm{Cl} 20,5 \%\end{array}$ \\
\hline 8 & $100 \% \mathrm{CH} 3 \mathrm{CN}$ & 1 & $\begin{array}{l}73 \% \text { aquoso, } \mathrm{CH}_{3} \mathrm{CN} \\
99,2 \% \text { e } \mathrm{MeOH} 0,8 \%\end{array}$ \\
\hline
\end{tabular}
não puderam ser recuperadas, devido à formação de azeótropo a $85^{\circ} \mathrm{C}$, nas amostras que continham $\mathrm{CH}_{2} \mathrm{Cl}_{2}$ após elevação do $\mathrm{pH}$ com $\mathrm{NaOH}$ houve a redução da proporção do $\mathrm{CH}_{2} \mathrm{Cl}_{2}$, mas não foi possível sua retirada total, as amostras em que só continham $\mathrm{CH} 3 \mathrm{CN}$ após destilação podem ser reutilizadas confirmada a ausência de água por espectro de infravermelho. Entrou-se em contato com os laboratórios que produzem esses resíduos e foi informado que as amostras que contenham $\mathrm{MeOH}$ e $\mathrm{CH}_{2} \mathrm{Cl}_{2}$ estas não poderiam ser reutilizadas para HPLC, este trabalho ainda se encontra em estudos para haver possível reutilização dessa $\mathrm{CH} 3 \mathrm{CN}$ com $\mathrm{MeOH}$.

Foi e está sendo realizado um trabalho piloto, junto com o Setor de Química Orgânica Experimental, no qual realizou-se um estudo nos polígrafos das aulas práticas das disciplinas de Química Orgânica Experimental I, QUI02223 e QUI02004 e foram identificadas as práticas que mais geram resíduos SOPP.

$\mathrm{Na}$ prática Extração de Óleos de Sementes Oleaginosas foi recuperado hexano, e na prática Extração da Cafeína foi recuperado diclorometano, ambos não precisaram de tratamento prévio e com apenas uma destilação consegue-se recuperar cerca de, $97 \%$ do volume dos mesmos, porém no caso do diclorometano o maior desafio é a estrutura das salas dos laboratórios, pois muito do volume que poderia ser recuperado é perdido no evaporador rotatório, o hexano já está sendo recuperado pelos técnicos do departamento.

Na prática Determinação do Coeficiente de Partição, pode ser recuperado álcool isoamílico, porém no semestre em que este projeto estava sendo implantado, devido a falta no estoque de álcool isoamílico os técnicos do setor tiveram que utilizar também o álcool n-amílico para as aulas práticas. Em ambos foi realizado pré-tratamento com secante $\mathrm{MgSO}_{4}$, seguido de destilação. O resultado obtido foi que o álcool isoamílico é uma mistura com 2-metilbutanol, resultado este confirmado por CG e RMN de $1 \mathrm{H} \mathrm{e} 13 \mathrm{C}$, na Figura 6 esta o cromatograma. 


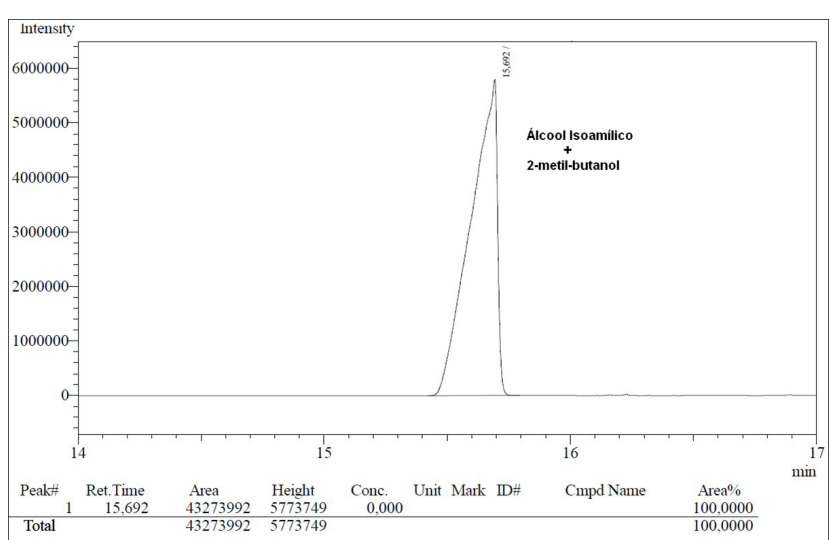

Figura 6. Cromatograma do álcool isoamílico.

Já na Figura 7 está o espectro de RMN de $1 \mathrm{H}$ no qual se pode calcular a proporção entre os alcoóis, o majoritário é álcool isoamílico na proporção de 5:1.

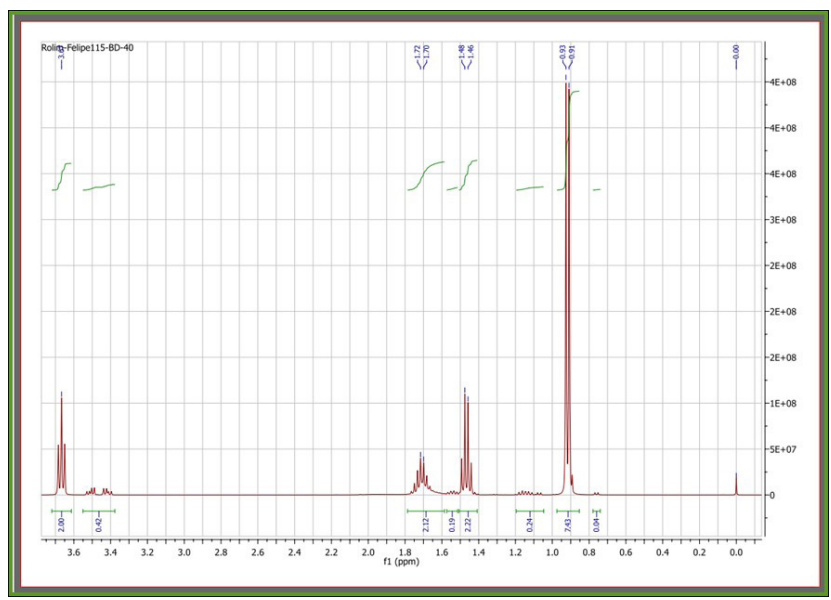

Figura 7. Espectro de RMN de $1 \mathrm{H}$ da mistura do álcool isoamílico com 2-metilbutanol.

O álcool n-amílico também se encontra com uma mistura do mesmo com o 2-metilbutanol, confirmado este resultado por $\mathrm{CG}$ no qual podemos observar na Figura 8, e RMN de $1 \mathrm{H}$ e 13C, o álcool majoritário é o n-amílico com proporção de 2:1.

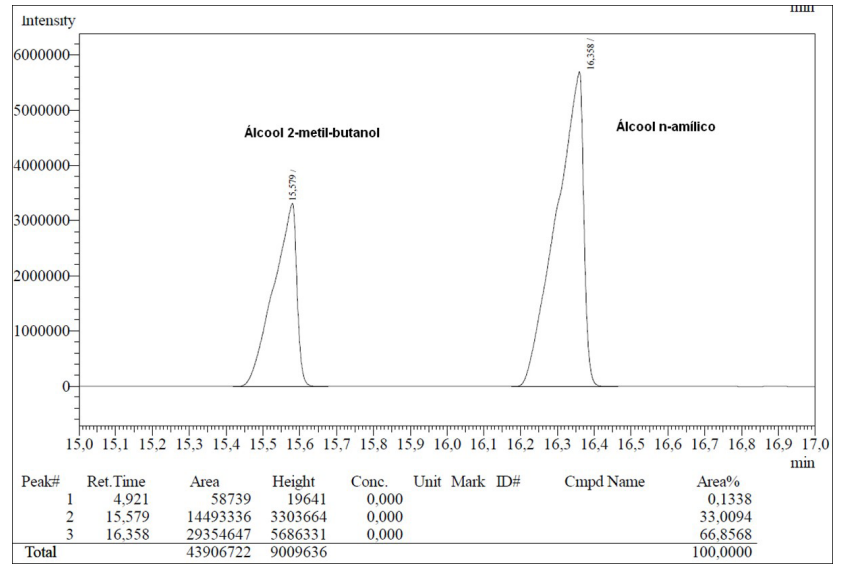

Figura 8. Cromatograma da mistura de álcool n-amílico com 2-metilbutanol.

\section{Conclusões}

Neste trabalho foram desenvolvidos métodos de recuperação e tratamento de 11 tipos de resíduos químicos diferentes, utilizados com frequência em muitos laboratórios e indústrias, e pode-se concluir que quanto menor for a mistura de solventes, maior a possibilidade e facilitação no processo de recuperação, em muitos casos com apenas uma destilação pode-se recuperar solventes como hexano, diclorometano, clorofórmio e metanol, como vistos neste trabalho com altos rendimentos e alto grau de pureza.

Há também resíduos que para serem reutilizados não precisam necessariamente a sua separação como é o caso do hexano e acetato de etila, que não podem ser separados pela formação de azeotrópo, porém estes são utilizados como mistura, então após a determinação da proporção entre eles, pode-se ajustar a mistura por diluição ou concentração a proporção a que se queira trabalhar.

Temos também o caso do etanol com polpa de caqui, no qual como o mesmo será reutilizado para extração com frutas não é necessária a secagem deste álcool, O resultado do xilol já está sendo implementado pelo CGTRQ, no qual os laboratórios que originam esse tipo de resíduo já o reutilizam e confirmam a eficiência. A acetonitrila que é um reagente de alto valor agregado, ainda se encontra em estudos devido aos diferentes perfis desse resíduo. 
O projeto piloto com o setor de graduação da Universidade está sendo expandido para recuperação de novos solventes utilizados em aulas práticas, e pretende-se se expandir esse trabalho a outros setores.

Com este trabalho podemos concluir que os resíduos químicos podem ser vistos de uma nova forma, pois muitos resíduos que seriam descartados podem ser reutilizados, muitas vezes de forma simples e econômica, evitando gastos na compra de novos reagentes e no descarte dos mesmos, este seria um método mais sustentável, pois evitaria o processo de incineração. Porém este é um trabalho que precisa ser continuo, sendo muito importante a conscientização dos geradores de resíduos químicos.

\section{Agradecimentos}

Gostaria de agradecer primeiramente meus pais por todo apoio familiar, ao professor orientador Eduardo Rolim de Oliveira que me passou muitos ensinamentos. Aos colegas do laboratório K210 e CGTRQ. A UFRGS e a PROPESQ.

\section{Referência Bibliográfica}

1. Alberguini, L.B.A. Quim. Nova, 26(2) 2003 Felippes, B.A. Revista de Ensino de Engenharia, v. 30, n. 2, p. 14-23, 2011 Jardim, W. de F. Quim. Nova, 21(5) 1998

\section{Bruna C. Dias*, Filipe W. Schwarz \& Eduardo R. de Oliveira}

${ }^{1}$ Instituto de Química, Universidade Federal do Rio Grande do Sul

*E-mail: bruninhaaa_cd@hotmail.com 